\title{
Augmenting Capsule Endoscopy Diagnosis: A Similarity Learning Approach
}

\author{
S. Seshamani ${ }^{1}$, R. Kumar ${ }^{1}$, T. Dassopoulos ${ }^{3}$, G. Mullin ${ }^{2}$, and G. Hager ${ }^{1}$ \\ 1 Department of Computer Science, Johns Hopkins University, Baltimore, MD \\ 2 Johns Hopkins Hospital, Baltimore, MD \\ 3 Washington University, School of Medicine St. Louis, MO*
}

\begin{abstract}
The current procedure for diagnosis of Crohn's disease (CD) from Capsule Endoscopy is a tedious manual process which requires the clinician to visually inspect large video sequences for matching and categorization of diseased areas (lesions). Automated methods for matching and classification can help improve this process by reducing diagnosis time and improving consistency of categorization. In this paper, we propose a novel SVM-based similarity learning method for distinguishing between correct and incorrect matches in Capsule Endoscopy (CE). We also show that this can be used in conjunction with a voting scheme to categorize lesion images. Results show that our methods outperform standard classifiers in discriminating similar from dissimilar lesion images, as well as in lesion categorization. We also show that our methods drastically reduce the complexity (training time) by requiring only one half of the data for training, without compromising the accuracy of the classifier.
\end{abstract}

\section{Introduction}

Wireless Capsule Endoscopy [1] is a non-invasive technology that is gaining popularity for diagnosis of Gastrointestinal (GI) diseases. The main advantage it provides, compared to traditional push endoscopy, is the increased comfort to the patient. The capsule endoscope, which is slightly larger than a common vitamin pill, contains lighting and imaging hardware, a wireless transmitter and a battery. It is taken orally by the patient and is propelled by peristalsis along the GI tract. Images are captured at the rate of $2 \mathrm{fps}$ by the device as it travels along the GI tract, and wirelessly transmitted to an archiving device that is attached to the patient's abdomen. The archiving device is then returned to the clinician who inspects the images (in the order of 50,000) to determine a diagnosis.

This diagnostic procedure, often 1-2 hours [1], requires the clinician to inspect several views of a pathology and evaluate it in a consistent manner. The low frame rate and lack of control of the imaging device, however, do not guarantee that subsequent images in the video sequence contain the same pathology.

\footnotetext{
* Supported in part by National Institutes of Health with Grant 5R21EB008227-02 and Johns Hopkins University internal funds.
} 
Advances in the area of computer vision and machine learning can be used to improve both time and consistency of this evaluation process. In this paper, we consider the particular case of improving the diagnosis of CD. CD is an inflammatory bowel disease which is characterized by discrete, well-circumscribed erosions and ulcers (lesions) in the small intestine (Fig. 1). During inspection, the clinician locates lesions and assigns severity labels to them. Upon completing evaluation of each lesion individually, the overall diagnosis is determined.

There are two ways in which this process can be improved. The first is by automatic matching for locating multiple views of a selected pathology. Seshamani et al. 2] propose a meta matching procedure that incorporates several simple matchers and a binary decision function that determines whether a pair of images are similar or not. The second diagnostic improvement is the enhancement of CD lesion scoring consistency with the use of a predictor which can determine the severity of the lesion based on previously seen examples.

Both of these problems can be approached from a similarity learning perspective. Learning the decision function for meta matching is a similarity learning problem 3. Lesion severity prediction is a multi-class classification problem which involves learning semantic classes of lesions based on appearance characteristics. Multi-class classification can also be approached from a similarity learning approach as shown in 34]. In this paper, we approach both of these problems as supervised pairwise similarity learning [5617] problems .

\section{Pairwise Similarity Learning}

The pairwise similarity learning problem is the following: Given a pair of data points, determine if these two points are similar, based on previously seen examples of similar and dissimilar points. A function that performs this task is called a pairwise similarity learner (PSL). A PSL is made up of two parts: a representation function, and a classification function. In addition, the PSL is also required to be invariant to the ordering of pairs. One method of assuring order invariance is by imposing a symmetry constraint on the representation function 2 . However, doing so can introduce a loss of dimensionality and possibly a loss of information that may be relevant for the classification task.

Order invariance of the PSL can also be ensured by imposing symmetry constraints on the classifier. We refer to such a classification function as a pairwise symmetric classifier. Several SVM-based pairwise symmetric classifiers have been proposed in the literature [5]6]. Within the SVM framework, symmetry is imposed by ensuring that the kernel function satisfies order invariance. In all prior work concerning pairwise symmetric classifiers, a pair is described by only one type of feature and the underlying assumption is that one distance metric holds for the entire set of points. However, this assumption may not hold when multiple features are used to describe data. The area of Multiple Kernel Learning [8910] has investigated several methods for combining features within the SVM framework.

In this paper, we present a novel pairwise similarity classifier for PSL using multiple features and nonsymmetric representations. 


\section{Mathematical Formulation}

Consider a pair of images $(I, J)$ and a set $\mathcal{X}$ consisting of $m$ image descriptors (features). Applying any $X_{i} \in \mathcal{X}$ to each image in the pair generates a representation $\tilde{x}=\left(x_{1}, x_{2}\right)$ where $x_{1}=\left\{X_{i}(I)\right\}$ and $x_{2}=\left\{X_{i}(J)\right\}$. A label $y \in\{1,-1\}$ is associated with each pair $\tilde{x}$, where $y=1$ implies a pair of similar images and $y=-1$ implies a pair of dissimilar images. The PSL problem can be written as follows: given a training set with $n$ image pair representations and their associated labels $\mathcal{T}_{m}=\left\{\left(\tilde{x}_{i}, y_{i}\right) \mid i=1 \ldots n\right\}$, compute a classifier $C$ that can predict the label of an unseen pair $\tilde{x}$ :

$$
C(\tilde{x})=C\left(\left(x_{1}, x_{2}\right)\right)=\left\{\begin{aligned}
1, & \text { if } \tilde{x} \text { represents a pair of similar images } \\
-1 & \text { otherwise }
\end{aligned}\right.
$$

Order invariance requires $C\left(\left(x_{1}, x_{2}\right)\right)=C\left(\left(x_{2}, x_{1}\right)\right)$. We refer to this as the pairwise symmetric constraint. An SVM trained on the set $\mathcal{T}$ would classify an unseen pair $\tilde{x}=\left(x_{1}, x_{2}\right)$ as:

$$
C(\tilde{x})=\sum_{\forall\left(x_{i}, y_{i}\right) \in \mathcal{T}} \alpha_{i} y_{i} K\left(\tilde{x}, \tilde{x}_{i}\right)+b
$$

where $b$ and $\alpha_{i}$ 's are learned from training examples and $K$ is a Mercer kernel. It is easy to note that this classifier will satisfy the pairwise symmetric constraint if $K$ satisfies: $K\left(\tilde{x}, \tilde{x}_{i}\right)=K\left(\left(x_{1}, x_{2}\right),\left(x_{i 1}, x_{i 2}\right)\right)=K\left(\left(x_{2}, x_{1}\right),\left(x_{i 1}, x_{i 2}\right)\right)$. We refer to such a kernel as a pairwise symmetric kernel (PSK).

\subsection{PSKs for One Descriptor}

Mercer Kernels can be generated from other Mercer Kernels by linear combinations (with positive weights) or elementwise multiplication [11. We use this idea to generate PSKs from simpler Mercer Kernels. Let us assume that we have two pairs: $\left(x_{1}, x_{2}\right)$ and $\left(x_{3}, x_{4}\right)$ and a base mercer kernel $K$, which can operate on a pair of points. A PSK (which operates on two pairs of points) can be computed by symmetrization of the base kernel. In [5], a second order PSK called the MLPK is introduced: $\hat{K}\left(\left(x_{1}, x_{2}\right),\left(x_{3}, x_{4}\right)\right)=\left(K\left(x_{1}, x_{3}\right)+K\left(x_{2}, x_{4}\right)-\right.$ $\left.K\left(x_{1}, x_{4}\right)-K\left(x_{2}, x_{3}\right)\right)^{2}$. This kernel is a linear combination of all second order combinations of the four base Mercer kernels. This kernel can be rewritten in terms of 3 PSKs as $\hat{K}=K_{1}+2 K_{2}-2 K_{3}$ where:

$$
\begin{aligned}
K_{1}= & K\left(x_{1}, x_{3}\right)^{2}+K\left(x_{2}, x_{4}\right)^{2}+K\left(x_{1}, x_{4}\right)^{2}+K\left(x_{2}, x_{3}\right)^{2} \\
K_{2}= & K\left(x_{1}, x_{3}\right) K\left(x_{2}, x_{4}\right)+K\left(x_{1}, x_{4}\right) K\left(x_{2}, x_{3}\right) \\
K_{3}= & K\left(x_{1}, x_{3}\right) K\left(x_{1}, x_{4}\right)+K\left(x_{1}, x_{3}\right) K\left(x_{2}, x_{3}\right)+ \\
& K\left(x_{2}, x_{4}\right) K\left(x_{1}, x_{4}\right)+K\left(x_{2}, x_{4}\right) K\left(x_{2}, x_{3}\right)
\end{aligned}
$$

The MLPK kernel is different from a second order polynomial kernel due to the additional base kernels it uses. A classifier trained with the MLPK kernel is comparable to a classifier trained with a second order polynomial kernel on 
double the amount of data (with pair orders reversed). SVM complexity can indeed be exponential in the number of training points (in the worst case) 12 . Secondly, a larger training dataset will generate more support vectors which increase run time complexity (classification time). Thus, the PSK is greatly beneficial in the reduction of both training and classification time.

\subsection{PSKs with More than One Descriptor}

For one descriptor, we obtain 3 second order $\operatorname{PSKs}\left(K_{1}, K_{2}\right.$ and $\left.K_{3}\right)$. So, given a set of $m$ descriptors, we can generate a total of $3 m$ second order PSKs: $Q=\left\{K_{i}^{\prime} \mid i=1 \ldots 3 m\right\}$. The problem now becomes the following: Given a set of $P S K$ s find a weight vector $d \in \Re^{3 m}$ that can generate a kernel $\hat{K}=\sum_{i}^{3 m} d_{i} K_{i}^{\prime}$ where $d_{i} \in d, K_{i}^{\prime} \in Q$. We use Simple Multiple Kernel Learning (SimpleMKL) 8 ] for automatically learning these weights. This method initializes the weight vector uniformly and then performs a gradient descent on the SVM cost function to find an optimal weighting solution. The proposed Generalized Pairwise Symmetric Learning (GPSL) training algorithm is outlined below.

Input: Training set $\mathcal{T}_{m}$ and $m$ base kernels.

Output: Weight Vector $d_{b e s t}$, SVM parameters $\alpha$ and $b$

- For each of the $m$ features, compute $K_{1}, K_{2}$ and $K_{3}$ (described in section 3.1) between all training pairs to generate the set $Q_{\text {train }}=\left\{K_{i}^{\prime} \mid i=1 \ldots 3 m\right\}$

- Apply SimpleMKL to find a weight vector $d_{b e s t}$.

- Learn the SVM parameters $\alpha$ and $b$ using a kernel generated as a linear combination of kernels in $Q$ using $d_{\text {best }}$.

To predict similarity of an unseen pair $\tilde{x}$ :

- Compute the set $Q_{\text {test }}$ using the test point and training examples.

- Generate a linear combination of these kernels using $d_{\text {best }}$

- Predict the similarity of the pair using the learned $\alpha$ and $b$.

\subsection{Multiclass Classification}

The multiclass classification problem for images is as follows: Given a training set consisting of $k$ images and their semantic labels $\mathcal{I}=\left\{\left(I_{i}, l_{i}\right) \mid i=1 \ldots k, l_{i} \in\right.$ $\{1 \ldots p\}\}$, where $I_{i} \mathrm{~s}$ are the images and $l_{i} \mathrm{~s}$ are the labels belonging to one of $p$ classes, compute a classifier that can predict the label of an unseen image I. From a similarity learning approach, this problem can be reformulated as a binary classification and voting problem: Given a training set of similar and dissimilar images, compute the semantic label of a new unseen image $I$. This requires two steps: 1) Learning similarities, and 2) Voting, to determine the label of an unseen image. We use the same method outlined in the GPSL algorithm above for similarity learning. Voting is then performed by selection of $n$ voters from each semantic class who decide whether or not the new image is similar or dissimilar to themselves. We refer to this algorithm as GPSL-Vote:

- Given $\mathcal{I}$, compute a new training set consisting of all combinations of pairs and their similarity labels: $\mathcal{T}=\left\{\left(\left(I_{i}, I_{j}\right)_{k}, y_{k}\right) \mid\left(I_{i}, l_{i}\right),\left(I_{j}, l_{j}\right) \in \mathcal{I}, y_{k} \in\right.$ $\{1,-1\}\}$ where $y_{k}=1$ if $l_{i}=l_{j}$ and $y_{k}=-1$ otherwise . 
- Train the GPSL using this set.

For a new image $I$,

- For each of the $p$ semantic classes, select $r$ representative images: $\left\{I_{1} \ldots I_{r}\right\}$ where $\left(I_{i}, y_{i}\right)$ is such that $y_{i}=p$. This generates a set of $q=p r$ images.

- Compute a set of pairs by combining each representative image with the new image $I:\left\{\left(I, I_{1}\right) \ldots\left(I, I_{q}\right)\right\}$

- Use the trained GPSL to predict which pairs are similar.

- For each semantic class, compute the number of similar pairs.

- Assign the new image $I$ to the class with the maximum number of votes.

\title{
4 Experimental Results
}

\subsection{Data Acquisition}

Our Capsule Endoscopy study repository contains 47 anonymized studies acquired with an approved Institutional Review Board (IRB) protocol. Each study may contain up to 8 hours of data acquired at 2 images per second. The CE study database contains annotated images and videos containing Crohn's Disease (CD) lesions manually selected by our clinical collaborators. In all our experiments, the size of the selected image is on the order of $150 \mathrm{X} 150$ pixels. All our experiments are performed using MATLAB.

\begin{abstract}
Annotated Lesion Data. Lesion annotation which was conducted by Dr. Dassopoulos involved selection of an image containing a lesion, and linking each image to a video segment consisting of 100-200 frames centered around the selected image. Each lesion image is then labelled as: Mild, Moderate or Severe (Fig. 1).
\end{abstract}
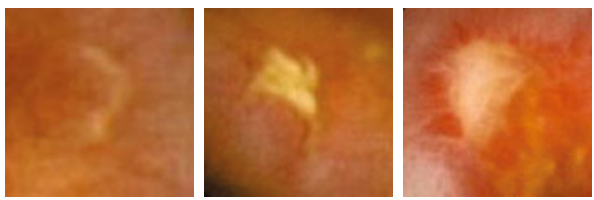

Fig. 1. Examples of Mild (left), Moderate (center) and Severe Crohn's Disease lesions

Annotated Matching Pairs. Each video sequence that is linked to a lesion image may contain multiple views of the same lesion. All views of the lesion within the video form a lesion set (Fig. 2). Various lesion sets contain between 2 and 25 image frames. We first manually segmented all regions containing lesions and then generated pairs within a lesion set as follows: Similar pairs were

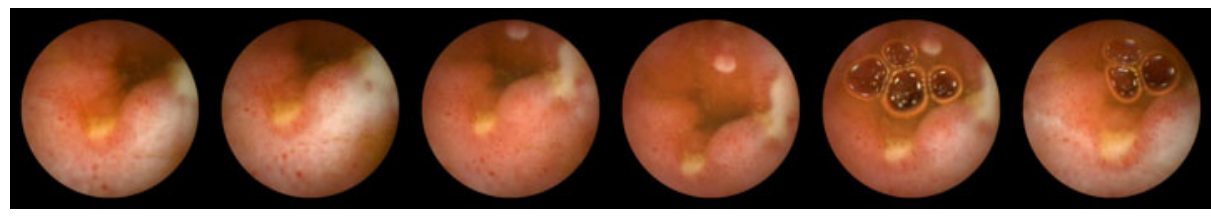

Fig. 2. A lesion set containing multiple views of the same lesion 
generated by pairing segmented lesion regions within the lesion set. Dissimilar pairs were generated by pairing segmented lesion regions with 150X150 regions sampled 60 pixels away from the annotated center of a segmented lesion.

\subsection{CE Lesion Matching Experiments}

The objective of these experiments is to show the effectiveness of our pairwise symmetric classifier for discriminating between similar and dissimilar $\mathrm{CE}$ lesion images. Here, a lesion is considered similar to another one only if it is a different view of the same pathology.

Pair representation: Each image in a pair was represented by a set of descriptors: MPEG-7 Homogeneous Texture Descriptors (HTD) [13, color weighted histograms (WH) and patch intensities (PI). WHs were generated by dividing the color space into 11 bins and populating a feature vector with points weighted by their distance from the image center. PIs were generated by dividing the image into 16 patches and populating a vector with the mean intensity in each patch. The number of histogram bins and patches were determined empirically. A nonsymmetric pair consists of two sets of these descriptors stacked together. For the symmetric reprsentation, descriptors element-wise squared difference was carried out between the two sets. A chi-squared base kernel was used for $\mathrm{WH}$ and a polynomial base kernel of order 1 was used for the other two descriptors in all experiments in this section.

Experiments: We first show the following experimentally: MLPK with a non-symmetric representation is better than using a nonsymmetric kernel with a symmetric representation. Our full data set contains 724 lesion image pairs. We used $30 \%$ of the data for testing and the rest for training of the HTD, $\mathrm{WH}$ and PI descriptors. Training of the HTD, WH and PI with the symmetric representation resulted in accuracies of: $52.2 \%$, $67.88 \%$ and $82 \%$ respectively. Training with the nonsymmetric representation resulted in
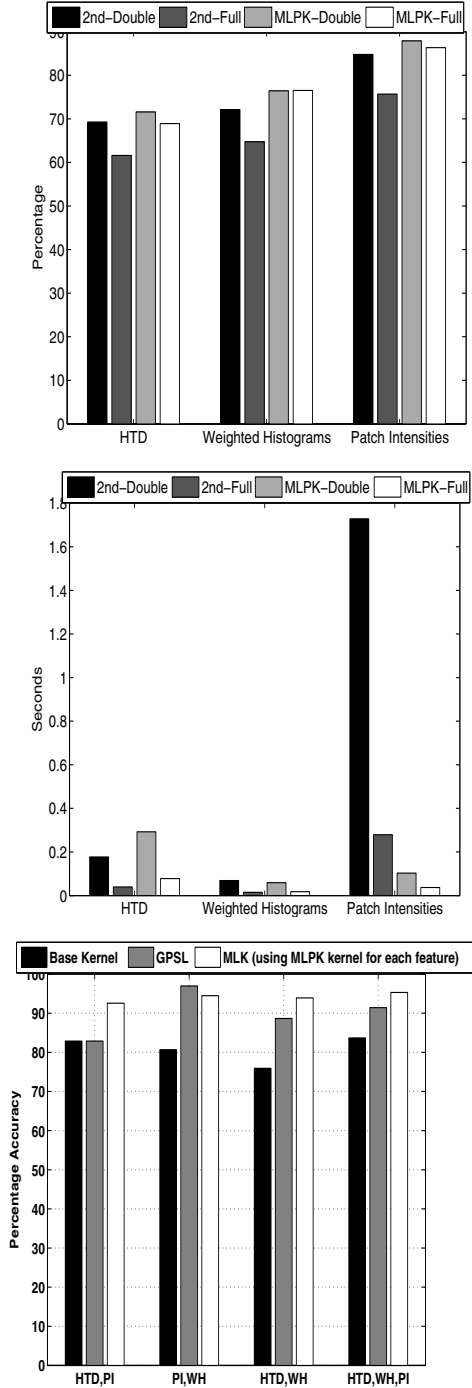

Fig. 3. Comparison of Accuracies (Top) and Training Time (Middle) for HTD, WH, PI using the full dataset and double dataset. Bottom: Comparison of the performance of the base kernel, MLPK and GPSL for various descriptor combinations 
accuracies of: $64.2 \%, 75.22 \%$ and $88 \%$ for $\mathrm{HTD}, \mathrm{WH}$ and PI respectively. Thus, we observe that generating a symmetric representation can cause a loss of information that is relevant for classification. Since MLPK is a second order kernel, the next question that arises is "How does an SVM trained with MLPK differ from an SVM trained with a second order polynomial kernel trained on double the data (generated by switching pair orders to enforce symmetry)?" To investigate this, we performed the following experiment. We first randomly selected $70 \%$ of the data as the full training set and the rest as the full testing set. We then doubled our data within each set by switching the order of pairs, to generate 1012 pairs for

Table 1. Four different experimental setups, varying in selection of a kernel and the amount of training data

\begin{tabular}{lll}
\hline Experiment & Kernel Type & Data \\
\hline \hline 2nd-Double & 2nd order poly & Double training set \\
\hline MLPK-Double & MLPK & Double training set \\
\hline 2nd-Full & 2nd order poly & Full training set \\
\hline MLPK-Full & MLPK & Full training set \\
\hline
\end{tabular}
the double training

set and 436 pairs for the double testing set. We performed classification (similar vs dissimilar pairs) using each descriptor 10 times by varying the type of training set and the type of kernel used (Table 1). Testing was always performed on the double testing set. Fig. 3 (bottom) shows the average accuracy and training time for all desriptors. The standard deviation of accuracies ranged from $2-4.5 \%$. We observe that the MLPK greatly drops the training time when half the data is used without compromising much on accuracy, unlike the second order polynomial kernel.

Finally, we show the effect of combining MLPKs generated from multiple features. We consider three algorithms for comparison: SVM with a base kernel, SimpleMKL using MLPK generated from the same base kernel (a total of $\mathrm{m}$ kernels) and GPSL (a total of 3m kernels also calculated from the same base kernel). We applied 5-fold CV to all three algorithms using all combinations of the three descriptors. Fig. 3 (bottom) shows the resulting accuracies. We observe that GPSL outperforms SVM with a base kernel in all cases. SimpleMLK with MLPK also performs much better than SVM with a base kernel in all cases, except the HTD descriptor.

\subsection{CE Lesion Classification}

Next, we considered the problem of classifying mild vs severe lesions (Fig. 1). A set of 10 lesions ( 5 in each class) were picked and three types of features were extracted: Haralick texture descriptor and Cross Correlation responses
Table 2. Accuracies of Different Classifiers used for lesion classification

\begin{tabular}{ll}
\hline Algorithm & Accuracy \\
\hline \hline SVM-Separate(Best Descriptor) & $72 \%$ \\
\hline SVM-MKL & $72 \%$ \\
\hline GPSL (Similarity learner) & $71.75 \%$ \\
\hline GPSL-Vote & $76 \%$ \\
\hline
\end{tabular}


of the blue and green bands with the same bands of a template lesion image. We then performed and compared three classification experiments: SVM with each descriptor separately (SVM-Separate) to directly classify lesion images, SVM with all features combined by SimpleMKL (SVM-MKL) to directly classify lesion images and finally with GPSL-Vote (which uses pairwise similarity learning). $\mathrm{CV}$ in all cases was performed on a "leave-two-out" basis, where the testing set was made up of one image from each class. All other images formed the training set. This generated a total of 25 total runs. In the case of GPSL-Vote, the similarity training dataset was generated using all combinations of pairs which are in the training set (totally 45 pairs). All pairs containing the two left-out images and a training image formed the test set. The results shown in Table 2 show the mean accuracy of all 25 runs. We observe that the SVM-MKL algorithm does only as well as the best classifier. However, GPSL-vote outperforms this, even for a small dataset with a small number of features.

\section{Conclusion}

We have demonstrated the use of pairwise symmetric learners for lesion matching and categorization. Our results show that the use of pairwise symmetric classifiers not only allows for nonsymmetric representation of data but also drastically reduces training time without compromising the accuracy of the classifier. We have also demonstrated the use of PSK's across multiple features for lesion match classification as well as semantic lesion classification in CE imagery. One extension of this work is the application of our classifier to a real-time matching system to increase the time efficiency of the clinician. Although the GPSL-vote experimentation was performed on a small dataset, the results are very encouraging. Some of the natural extensions of this work include experimentation with more descriptors and larger datasets for GPSL-vote.

\section{References}

1. Iddan, G., Meron, G., Glukhovsky, A., Swain, P.: Wireless capsule endoscopy. Nature 405(6785), 417 (2000)

2. Seshamani, S., Rajan, P., Kumar, R., Girgis, H., Mullin, G., Dassopoulos, T., Hager, G.: A meta registration framework for lesion matching. In: Yang, G.-Z., Hawkes, D., Rueckert, D., Noble, A., Taylor, C. (eds.) MICCAI 2009. LNCS, vol. 5761, pp. 582-589. Springer, Heidelberg (2009)

3. Chen, Y., Garcia, E.K., Gupta, M.R., Rahimi, A., Cazzanti, L.: Similarity-based classification: Concepts and algorithms. JMLR 10, 747-776 (2009)

4. Cazzanti, L., Gupta, M.R.: Local similarity discriminant analysis. In: ICML (2007)

5. Vert, J.P., Qiu, J., Noble, W.S.: A new pairwise kernel for biological network inference with support vector machines. BMC Bioinformatics 8(S-10) (2007)

6. Kashima, H., Oyama, S., Yamanishi, Y., Tsuda, K.: On pairwise kernels: An efficient alternative and generalization analysis. In: Theeramunkong, T., Kijsirikul, B., Cercone, N., Ho, T.-B. (eds.) PAKDD 2009. LNCS, vol. 5476, pp. 1030-1037. Springer, Heidelberg (2009) 
7. Oyama, S., Manning, C.D.: Using feature conjunctions across examples for learning pairwise classifiers. In: Boulicaut, J.-F., Esposito, F., Giannotti, F., Pedreschi, D. (eds.) ECML 2004. LNCS (LNAI), vol. 3201, pp. 322-333. Springer, Heidelberg (2004)

8. Rakotomamonjy, A., Bach, F.R., Canu, S., Grandvalet, Y.: Simplemkl. JMLR 9 (2008)

9. Varma, M., Babu, B.R.: More generality in efficient multiple kernel learning. In: ICML, pp. 1065-1072 (June 2009)

10. Gehler, P., Nowozin, S.: Let the kernel figure it out: Principled learning of preprocessing for kernel classifiers. In: CVPR (2009)

11. Cristianini, N., Shawe-Taylor, J.: An Introduction to Support Vector Machines: and Other Kernel-Based Learning Methods. Cambridge University Press, Cambridge (2000)

12. Gärtner, B., Giesen, J., Jaggi, M.: An exponential lower bound on the complexity of regularization paths. In: CoRR (2009)

13. Manjunath, B., Ohm, J., Vasudevan, V., Yamada, A.: Color and texture descriptors. IEEE CSVT 11(6), 703-715 (2001) 